\title{
Bering - The first deep space mission to map asteroidal diversity, origin and transportation
}

\author{
Anja C. Andersen ${ }^{1}$, René Michelsen ${ }^{2}$, Henning Haack ${ }^{3}$, and John L. Jørgensen ${ }^{4}$ \\ ${ }^{1}$ NORDITA, Blegdamsvej 17, 2100 Copenhagen, Denmark, E-mail: anja@ nordita.dk \\ ${ }^{2}$ Astro. Obs., NBIfAFG, Juliane Maries Vej 30, 2100 Copenhagen Denmark, E-mail: rene@astro.ku.dk \\ ${ }^{3}$ Geological Museum, Øster Voldgade 5-7, 1350 Copenhagen K, Denmark, E-mail: hh@ savik.geomus.ku.dk \\ ${ }^{4}$ Ørsted*DTU, MIS, Building 327, Tech. Uni. of Denmark, 2800 Lyngby, Denmark, E-mail: jlj@ oersted.dtu.dk
}

\begin{abstract}
Asteroids are remnants of the material from which the Solar System formed. Fragments of asteroids, in the form of meteorites, include samples of the first solid matter to form in our Solar System 4.5 mia years ago. Spectroscopic studies of asteroids show that they, like the meteorites, range from very primitive objects to highly evolved small Earthlike planets that differentiated into core mantle and crust. The asteroid belt displays systematic variations in abundance of asteroid types from the more evolved types in the inner belt to the more primitive objects in the outer reaches of the belt thus bridging the gap between the inner evolved apart of the Solar System and the outer primitive part of the Solar System. High-speed collisions between asteroids are gradually resulting in their break-up. The size distribution of kilometer-sized asteroids implies that the presently un-detected population of sub-kilometer asteroids far outnumber the known larger objects. Sub-kilometer asteroids are expected to provide unique insight into the evolution of the asteroid belt and to the meteorite-asteroid connection. We propose a space mission to detect and characterize sub-kilometer asteroids between Jupiter and Venus. The mission is named Bering after the famous navigator and explorer Vitus Bering. A key feature of the mission is an advanced payload package, providing full on board autonomy of both object detection and tracking, which is required in order to study fast moving objects in deep space. The autonomy has the added advantage of reducing the cost of running the mission to a minimum, thus enabling science to focus on the main objectives.
\end{abstract}

\section{Introduction}

Our present understanding of asteroids and their orbits is almost entirely based on surveys of main-belt asteroids with diameters larger than $10 \mathrm{~km}$. Ground based telescopes cannot detect smaller objects except within the immediate vicinity of Earth and no spacecraft has, so far, detected any previously unknown asteroids. Despite the fact that several spacecrafts to date, statistically, must have passed by smaller asteroids, the technology employed in these vessels has not held the capability of detecting these objects. Therefore such encounters have gone unnoticed by. Recent development in the autonomy of space-borne image-

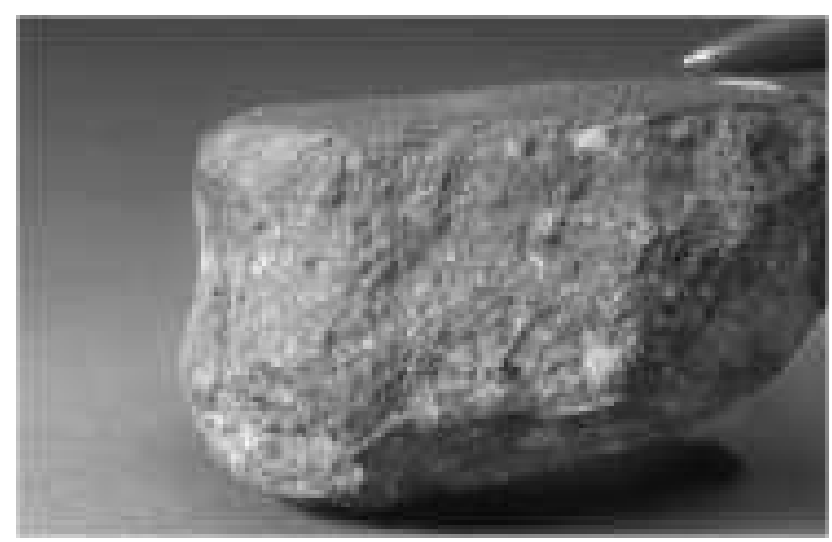

Figure 1. A fragment of the carbonaceous chondrite Allende that fell in Mexico in 1969. The meteorite is composed of dark fine grained dust, mm-sized spherical inclusions (chondrules) and white inclusions know as calciumaluminum-rich inclusions (CAIs). The CAIs formed 4567 My ago and are the oldest known solids formed in the Solar System. Carbonaceous chondrites probably originate from C-type asteroids which are common in the outer main-belt.

and computer-technology has changed this, so that it is now possible to detect, classify and observe during an encounter with a small asteroid.

The sub-kilometer objects between Jupiter and Venus, in particular the Near-Earth Asteroids (NEAs), are expected to fill the gap between the meteorites that we have studied in very great detail in the laboratory and their large parent asteroids in the main belt that may be studied with Earthbased telescopes. The meteorites have been knocked off their parent asteroids through impacts. These impacts delivered fragments in a large range of sizes. Streams of small asteroids are connected to parent asteroids via dynamical mechanisms responsible for the transfer of material to the inner Solar System [1].

Meteorites are highly diverse geological samples of asteroids, the Moon and Mars. They range from very primitive samples of the first solids to form in the Solar System (Fig. 1) to highly evolved samples of differentiated planetary objects. The latter include iron meteorites from asteroid metal 
cores resembling the core of the Earth and basaltic meteorites from the surfaces of asteroids that had an active volcanic activity more than 4 billion years ago. Studies of meteorites provide detailed information about the chronological, geochemical and geological evolution of the early Solar System. But unlike geological samples from the Earth, meteorites are delivered without any information about the setting of the sampling site. Small asteroids, which represents fragments of asteroid collisions in the recent past, probably have fresh surfaces with minimal regolith cover and with minimal exposure to cosmic rays, hence with a surface that is more representative of the interior. Fragments in the form of meteorites may therefore more easily be linked to small asteroids than large asteroids with highly evolved surface properties.

The small asteroids are therefore vital for our understanding of mass transportation in the inner Solar System, as well as for providing a firm basis for the dynamical and physical relation between meteorites, NEAs and the asteroid main belt. For a thorough discussion on asteroid research see the book by Bottke et al. [2].

The Bering mission will consist of two fully autonomous spacecrafts which detects the asteroids, determine their orbital parameters, light curve and spectral characteristics. The two spacecrafts will be identical and fly in a loose formation. The spacing between the two probes make it possible to determine the orbital parameters of the asteroid. Each probe will be able to provide autonomous detection, tracking, mapping and ephemeris estimation of asteroids. The autonomous instrumentation also include automatic linkup with Earth and inter spacecraft communication. The autonomous operations of the instruments are centered on the Advanced Stellar Compass [3].

The Bering mission will consist of two fully autonomous spacecrafts which detects the asteroids, determine their orbital parameters, light curve and spectral characteristics. A laser ranger will be used to keep track of the relative positions of the two spacecraft. Simultaneous observations from both spacecraft will allow us to accurately determine the distance to detected objects and thus make it possible to determine the orbital parameters of objects that are quickly passing out of view. The autonomous instrumentation also include automatic linkup with Earth and inter spacecraft communication. The autonomous operations of the instruments are centered on the Advanced Stellar Compass, cf. [3], [4] and [5].

\section{Asteroids and meteorites}

We have very little information on the abundance and characteristics of objects smaller than about $1 \mathrm{~km}$ except for those that have been observed in the immediate vicinity of Earth. The power law distribution of asteroid sizes suggests that objects smaller than $1 \mathrm{~km}$ are very abundant, see Fig. 2 .

Within the asteroid belt we have no information about these objects, since they cannot be observed from Earth and no spacecraft have been actively looking for them.

The orbits of these small objects can be perturbed by physical processes in the asteroid main belt, such as collisions. Since any fragmentation process tends to generate power law size distributions of the fragments we should not be surprised to see that the size distribution of asteroids follow

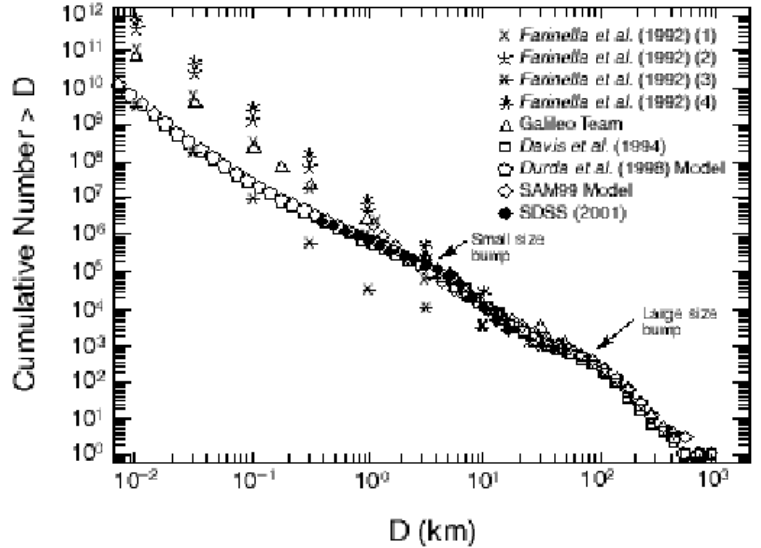

Figure 2. Nine different estimates of the main-belt asteroid size distribution. The small size distribution is obtained by extrapolating the observed large size trends. Figure taken from [6].

a power law distribution. There is, however, reason to believe that the very smallest asteroids are less abundant in the main belt than a simple extrapolation of the data from the larger asteroids would suggest. Smaller asteroids are more easily influenced by the Yarkovsky effect ${ }^{1}$ [7] and may thus be removed from the belt on a shorter time scale than the larger asteroids. Still smaller fragments may be removed as a consequence of the Poynting-Robertson effect ${ }^{2}$. A direct measurement of the size distribution would allow us to gain evidence of these physical mechanisms, and how they may have influenced the development of the main belt.

Meteorites are fragments of approximately 150 different main belt asteroids. Since meteorites are well studied representatives of the abundant low mass tail of the objects that impact on Earth, a better understanding of their origin in the asteroid belt and their subsequent orbital evolution will allow us to better understand the transfer of objects from the main belt to the NEA population. Detailed studies of meteorites allow us to determine age constraints for the disruptions of their parent asteroids and the subsequent transfer of fragments to the inner Solar System.

Asteroid photometry shows that asteroids are very diverse in terms of surface mineralogy. The variation is equivalent to the variation observed among meteorites although a near exact match between the spectrum of an asteroid and a group of meteorites is extremely difficult to find. Possible reasons for the differences between reflectance spectra of asteroids and meteorites include fine grained dust cover on asteroids, micro meteorite impacts on asteroids and exposure to cosmic radiation. Changes of the asteroids reflectance spectrum due to these poorly characterized processes are referred to as space weathering. Only in one case has it been possible to establish a reasonably good case for

\footnotetext{
${ }^{1}$ The effect of its rotation on the path of a small object orbiting the Sun. Rotation causes a temperature variation, so thermal energy is re-radiated anisotropically.

${ }^{2}$ The effect of solar radiation on small objects orbiting the Sun, which causes them to spiral slowly in. The object absorb solar energy that is streaming out radially, but re-radiate energy equally in all directions. As a consequence there is a reduction in the kinetic energy, and thus in orbital velocity, which has the effect of reducing the size of the orbit.
} 


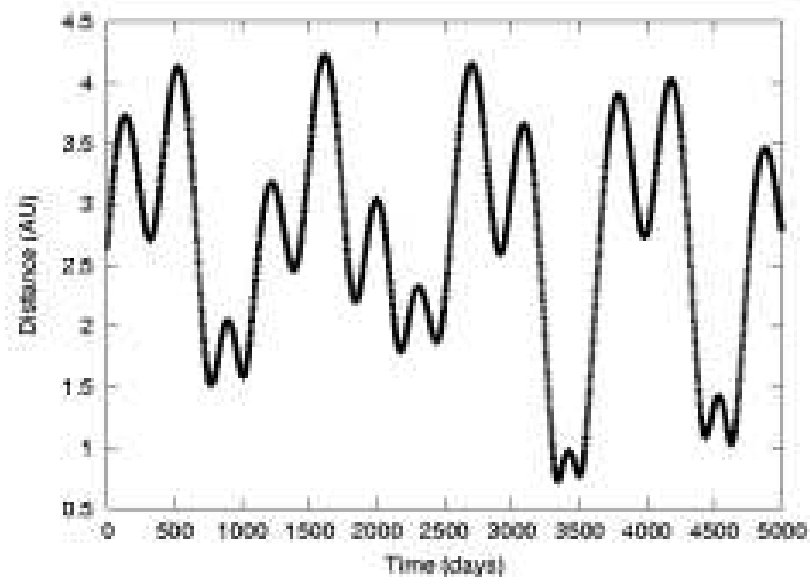

Figure 3. Variations in the distance to Earth as a function of time of the Near-Earth Asteroid 2002 NY31. Epoch of the figure is June 10, $2003=$ JD 2452800.5.

a specific asteroid-meteorite relationship. The unique spectrum of the basaltic surface of 4 Vesta makes it the prime candidate for about 400 igneous meteorites known as the HED meteorites. There is considerable interest in establishing links between other groups of meteorites and their parent asteroids.

Unlike the larger asteroids studied from space so far, small objects are expected to have young surfaces, and their surfaces are therefore representative of their interior. A longstanding debate has been the relationship between the silicaceous (S-type) asteroids and the ordinary chondrite meteorites. Differences in spectral characteristics have been attributed to a poorly constrained space weathering process. Since small asteroids probably have smaller life times and less gravity we should expect them to have younger surfaces that have been exposed to space weathering for a shorter period of time. Also the lower gravity should reduce the build-up of a regolith cover on their surfaces that may hide geologic units underneath. Both of these effects will make a comparison with spectral characteristics of meteorites and other materials easier. Data on the orbital distribution of objects with spectral characteristics similar to a group of meteorites may provide new constraints on the meteoriteasteroid relationship.

\section{Detection of asteroids}

A number of things distinguish the brightness of an asteroid from that of a distant star. Where the emitted radiation from a star is due to internal nuclear processes, the brightness of an asteroid entirely depends on reflected sunlight in terms of the illuminated area, as well as the albedo. This implies a dependency on the distances asteroid-observer and asteroid-Sun as well as the phase angle. In total 5 parameters are needed to describe the brightness variations, of which 3 parameters have an explicit time dependency. Thus, even for a constant distance between an observer and the asteroid, the brightness will vary due to the changing distance to the Sun, quite a different situation from observing remote stars. In addition, asteroids are objects moving with velocities of the same order of magnitude as the Earth, with distances to the Sun at the same order of magnitude

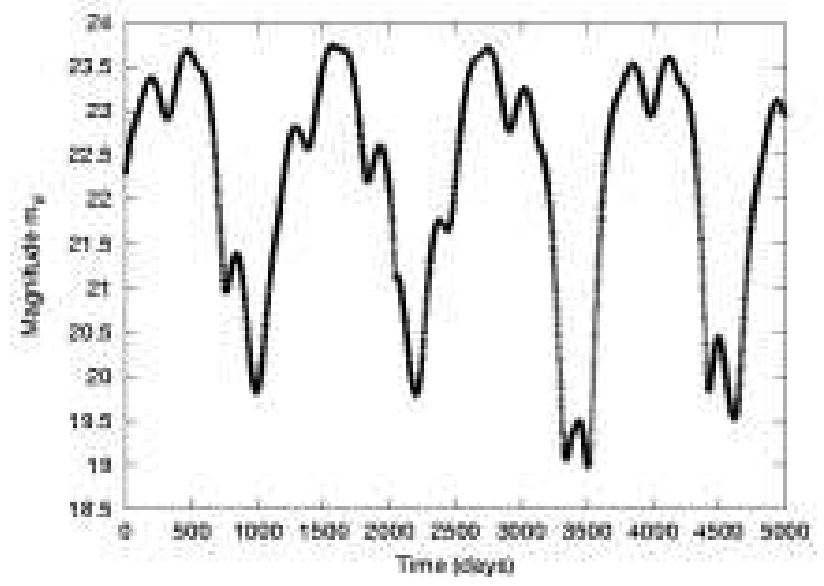

Figure 4. Variations in the $V$-magnitude $\left(m_{V}\right)$ as a function of time of the Near-Earth Asteroid 2002 NY31 as seen from Earth. Epoch of the figure is June 10, $2003=J D 2452800.5$. The absolute magnitude of this object is $H=17.3$, corresponding to a diameter of around $1 \mathrm{~km}$.

as the distance Sun-Earth. This introduces brightness variations, which are not present for distant self-luminous objects. Examples of these variations are illustrated in Figs. 3 and 4

Due to the eccentricity of $e=0.55$ of 2002 NY31, the distance to the Earth shows a very large variation over time, in terms of repeated periodic variations. This behavior directly influence the $V$-magnitude $\left(m_{V}\right)$ as seen from Earth. It is seen (Fig. (4) that the brightness peaks seems to fall out from a faint background magnitude, and that only within constrained regions is the object observable from a telescope with a given magnitude limit.

These variations are dependent on the mentioned physical and geometric parameters, so the smaller the objects, the more restricted are the favorable periods of observability. An example ${ }^{3}$ of this can be found in Figs.5 and 6

The synthetic object in these figures has an absolute magnitude of $H=32.7$, corresponding to a diameter of around $1 \mathrm{~m}$. It is seen, that upon a close approach to the Earth, the magnitude decreases drastically, from a background level above $m_{V}=30$ to a sudden brightness of around $m_{V}=15$. It is also noticed that the brightness peak is very sharp, in fact the object magnitude is below $m_{V}=20$ for merely 4.8 hours. For a telescope with a given magnitude limit, these objects are only observable during the occurrence of such a brightness peak, unless the telescope is able to reach very faint magnitudes i.e. $m_{V}=30$. In addition, the brightness peak must appear while the object is within the field of view of the observer. In practice, a survey telescope must constantly monitor the whole sky in order not to miss the object due to the short time of visibility.

An obstacle, compared to the traditional way of making ground based observations, is that during one night, each field of sky will typically only be imaged one time as a series of three or more short exposures, and the reduction of

\footnotetext{
${ }^{3}$ The synthetic object has the orbital parameters $a=1.00583363, e=$ $0.04361874, i=23.00978088, \Omega=29.24312401, \omega=89.19306946$, $M=67.975616464$, epoch $=J D 2452000.5, H=32.70$, slope parameter $G=0.32$.
} 


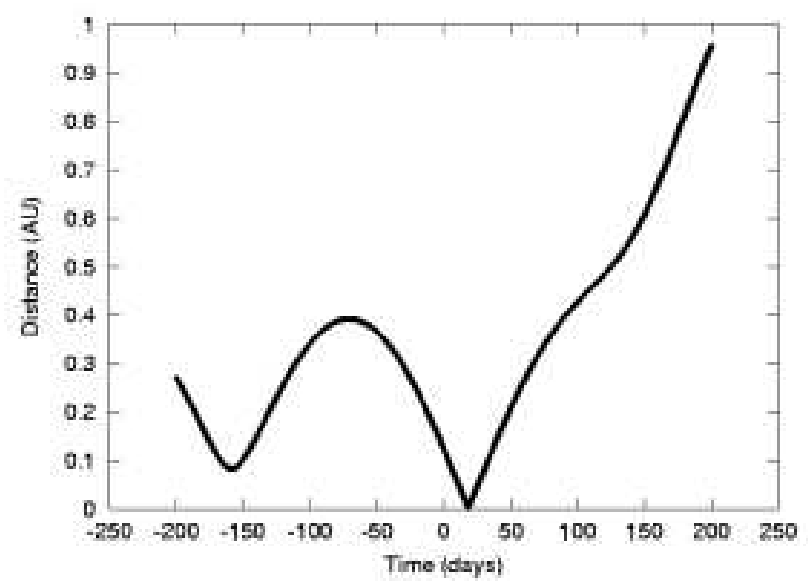

Figure 5. Distance to Earth of a synthetic NEA over 400 days. The resolution is 0.02 days $=28.8$ minutes. The figure was obtained using modifications to the SWIFT integrator [8], see also [9] for more details.

the images will be done during the following day or days. Due to the transient nature of the brightness peak of the small asteroids, the image reduction would however have to be done real time, in order to detect the object immediately, and initiate follow-up observations for a verification. A ground based fast-response survey has been proposed [10], however with a per-night data reduction it is still remote from a real time solution.

There is an additional obstacle that must be overcome. For sub-meter objects, the brightness peaks only occurs upon very close approach to the observer. This means, that the angular velocity relative to the observer becomes very large, for the shown example it is of the order of a few hundred "/sec. A ground based observer, using a survey telescope, will typically need to make an optimization of the exposure time involving the pixel size, the seeing, the astrometric accuracy and the angular velocity of the objects of interest. Typically, a survey using a large telescope going to faint magnitudes, will make use of a series of $60 \mathrm{~s}-120 \mathrm{~s}$ exposures. Due to the large radial velocity, the signal from the object will be smeared out on the detector (trailing loss).

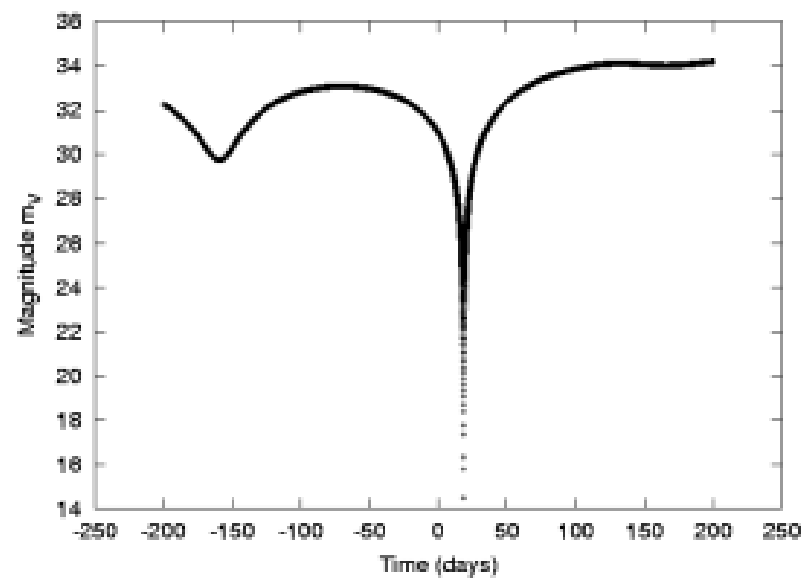

Figure 6. Variations in the V-magnitude $\left(m_{V}\right)$ of a synthetic NEA as seen from Earth. See Fig.5 and text for details.
In fact, in order to reach a resolution around 1" for the fast-moving objects, a typical survey telescope would be restricted to exposure times around a hundredth of a second, presumably posing heavy demands regarding the size of the telescope.

Outside the brightness peak, the object is moving with an angular velocity of a few "/min, so even for a telescope able to reach $m_{V}=30$, the exposure time would be constrained to $60-120 \mathrm{~s}$.

In summary, a survey telescope trying to detect these small asteroids must be capable of an all-sky monitoring, able to perform real time data analysis, and able to handle fast moving objects. The alternative would be a telescope able to reach $m_{V}=30$, or beyond, with exposure times per image frame limited to a few minutes. For the latter option, our conjecture is that such a telescope is currently not technologically feasible. For the first option, we propose Bering as a solution to meet these requirements in terms of the autonomy and the application of the Advanced Stellar Compass. In addition to the simple object detection, discussed in the above, Bering also provides the possibility of performing spectroscopy/photometry of the feasible objects, thus adding another dimension of performance in comparison to ground based observations.

\section{Mission profile requirements}

For the reasons outlined above, the main goal of the proposed Bering mission is to detect and characterize a sizable amount of sub-kilometer objects from space. This will be the first systematic survey of sub-kilometer objects in the Solar System. The numbers detected need to be sufficiently high that the distribution of small objects with similar spectral characteristics and therefore potentially identical parent asteroid may be established throughout the main belt.

Due to the transient visibility variations, the probes must detect the objects and guide the science instruments in a fully automated process.

The two spacecrafts will each carry an Advanced Stellar Compass (ASC) system with 7 camera heads, a folding mirror based multi-spectral telescope imager, magnetometers and a laser-ranger. These instruments will make it possible to obtain orbital parameters, light curves, rotation state, surface composition, and in some cases albedo, size, high quality images, mass and magnetic properties, cf. [12].

In order to determine the distribution and dynamics of small objects and their links to the NEA population we need to detect objects in the main asteroid belt as well as inside the Earths orbit. The objects within the mean motion resonances in the asteroid belt that are either already NEAs or are becoming NEAs within a short time frame, generally have aphelion within the main belt and spend most of their time outside the Earth orbit. We therefore propose a mission profile that would give us data on the distribution of small objects all the way from $0.7 \mathrm{AU}$ to $3.5 \mathrm{AUs}$, see Fig.7 Such an orbit can be achieved with a single unpowered gravity assist maneuver from Venus whereas the $\Delta \mathrm{V}$ required to reach Venus could be delivered directly by the launcher, which could be of the Soyuz class.

For each detected object we propose to automatically determine: 


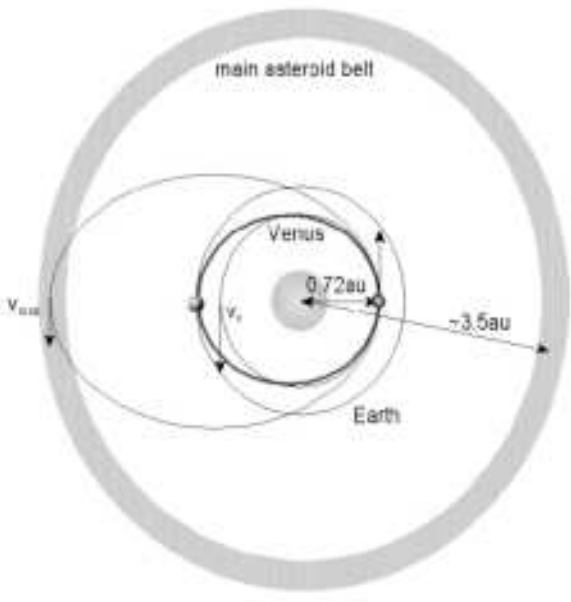

Figure 7. Mission profile. Planet and asteroid orbits are shown circular and coplanar for simplicity. The figure also show the positions of Earth and Venus during the first phase of the mission and it assumes a fly-by at the second passage of the perihelion (1.5 period).

- The heliocentric position, and velocity vector of the object.

- Multicolor photometry of the reflected light from the object in the 350-2200 $\mathrm{nm}$ range.

- The light curve of the object and thereby its rotation period.

For a few selected larger objects we furthermore propose to:

- Record multicolor images of the surface.

- Determine the mass and magnetic moment of the object.

The data will allow us to determine a current orbit for the object. With a detection at $m_{V}=9$ for the Advanced Stellar Compass and a detection limit of $m_{V}=25$ for the multicolor imager we will be able to follow the object out to a distance of approximately 1500 times the distance where it was detected. Depending on the geometry and the size of the object this will typically allow us to follow the object for days to weeks and determine high precision orbital parameters for a large fraction of the orbital arc. The orbital data will also allow us to determine the objects position in orbital space and its proximity to resonances and/or other asteroids or asteroid families with similar spectral characteristics and dynamical characteristics.

The photometry will allow us to determine the spectral type of the object. This will make it possible for us to determine its relationship with other asteroids and/or groups of meteorites with similar mineralogy. Ultimately, we will attempt to backtrack the object to its parent asteroid

The ability to detect objects down to $m_{V}=25$ from within the asteroid belt with the multicolor imager may also be utilized to further constrain the density of small objects. In a few campaigns we propose to make a series of frames with either subsequent on board processing or data processing on Earth. This will allow us to determine the number of asteroidal objects in each frame, going to very faint objects. Although the size-distance relationship cannot be determined on the basis of a few frames these data may be used to check predictions based on models of the distribution of asteroids.

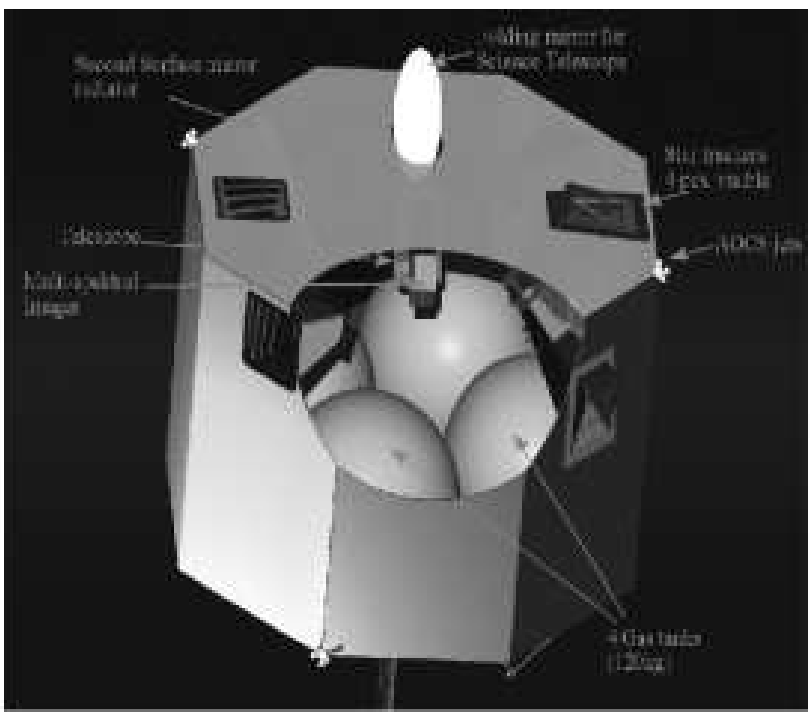

Figure 8. Artistic impression of the Bering preliminary design.

\section{Object detection rate}

The requirement to the number of objects Bering would detect per unit of time is important to quantize both due to the scientific feasibility estimate, as well as to formulate the design requirements of Bering. Due to the complex variations in brightness, this is not trivial to estimate.

For an observer moving in an inertial frame, it would maybe be possible to estimate the flux of objects through the detection sphere of Bering. However, due to the proper motion of Bering, and due to the strong dependency on geometry, it becomes a more complex task to evaluate the flux of objects. A possible approach could be to apply the estimates of collision rates in the asteroid belt [11], however for a detailed examination of the Bering mission profile, we need a more direct approach [9].

\section{Spacecraft design}

The Bering spacecrafts have to possess a high degree of autonomy since at a distance of $3 \mathrm{AU}$ the closed loop to Earth is about $48 \mathrm{~min}$. The autonomy must encompass all everyday routines, such as object detection, classification, tracking and initial data acquisition. An overview of the payload is provided in Table 1

The core autonomy of the proposed mission is centered on the Autonomous Stellar Compass (ASC), a fully autonomously operation star tracker [3]. The ASC consist of a powerful microcomputer with search engine and star catalog, with several camera heads attached, typically two to four. Each camera head will acquire an image of the night sky in the Field Of View (FOV) two times per second and pass on the digital image to the data processor, see [5].

Since all luminous objects above the selected threshold are detected, non stellar such as galaxies, nebulae, other satellites and asteroids are also autonomously picked out. They are identified as luminous objects brighter than the threshold, with no matching object in the star catalog. Since the ASC has established the attitude of the image in question, the apparent position of the object is established with high 
Table 1. Scientific payload.

\begin{tabular}{|l|l|l|}
\hline Instrument & Science & $\begin{array}{l}\text { Mission } \\
\text { support }\end{array}$ \\
\hline \hline $\begin{array}{l}\text { Advanced } \\
\text { Stellar } \\
\text { Compass }\end{array}$ & $\begin{array}{l}\text { Detection of } \\
\text { asteroids }\end{array}$ & $\begin{array}{l}\text { Additude and } \\
\text { navigation }\end{array}$ \\
$\begin{array}{l}\text { Multiband } \\
\text { Imaging } \\
\text { System }\end{array}$ & $\begin{array}{l}\text { Surface composition, } \\
\text { rotation and spatial } \\
\text { extend of asteroid, } \\
\text { accurate apparent } \\
\text { position of target }\end{array}$ & $\begin{array}{l}\text { Location } \\
\text { of sister } \\
\text { spacecraft }\end{array}$ \\
$\begin{array}{l}\text { Laser } \\
\text { Ranger }\end{array}$ & $\begin{array}{l}\text { Exact distance to } \\
\text { close asteroids }\end{array}$ & $\begin{array}{l}\text { Ranging of } \\
\text { sister space- } \\
\text { craft }\end{array}$ \\
$\begin{array}{l}\text { Magnetometer } \\
\text { Probes }\end{array}$ & $\begin{array}{l}\text { Magnetic properties, } \\
\text { gravity estimate of } \\
\text { asteroid and thereby } \\
\text { mass and density }\end{array}$ & $\begin{array}{l}\text { Solar } \\
\text { pressure } \\
\text { estimation }\end{array}$ \\
\hline
\end{tabular}

accuracy. Typically the instant position determination is in the range of 3 arc seconds, but since the ASC update the measurement per camera twice per second, averaging will bring the accuracy to the sub-arc second level in a matter of seconds. In this way, the entire FOV is searched for nonstellar objects. Since the FOV covers $1 \%$ of the entire night sky, a scanning operation of the spacecraft will have to be employed. We plan to arrange 7 camera heads on board the spacecraft, whereby almost $100 \%$ sky coverage is guaranteed at a spin rate of once per hour.

The non-stellar objects are entered into a database. When the same area of the night sky is revisited after one rotation period of the spacecraft, the apparent position of the objects are established again. Any object thus showing a proper motion above the measurement noise is moved to an asteroid candidate list for further investigations [13].

The main science instrument on the proposed mission is a multi-band imaging telescope, see Fig. 8 and [5]. The telescope might have a focal length of $1 \mathrm{~m}$, and an entrance pupil of about $0.3 \mathrm{~m}$. To compensate for the spacecraft rotation and to allow for fast tracking, the telescope is fitted with a folding mirror. To enable fast and accurate tracking of a target, the telescope is furthermore equipped with an ASC camera, mounted on the back of the secondary mirror. The ASC camera and the telescope optical axes are approximately parallel. From simultaneous images of the night sky, the relative orientation of the telescope to the ASC camera is easily established with high accuracy.

When the ASC has detected a Sun orbiting body, the apparent inertial position of the body is measured and passed on to the main onboard processor, that at a convenient time, points the telescope at the target. This pointing is greatly facilitated by the ASC camera that is co-aligned with the telescope,cf. [12] and [5]. The multi-spectral imager serves multiple functions too. Its main objective is to character- ize the target through spectral analysis and to obtain high fidelity multispectral science images. However, through the guide information from the ASC, the stars in the telescope image are analyzed, whereby an extremely accurate apparent position of the target is found. This procedure allows the Bering spacecrafts to keep track of more than 1000 objects at any give time of the mission.

The laser ranger will determine the distance to all objects that happen to pass close by the spacecraft. Small freeflying magnetometer probes will be launched toward selected larger objects that comes within close range of the spacecrafts. At close encounter the magnetometers will provide information about the distribution of the magnetic field, and as such probe the interior of the asteroid. The trajectory of the magnetometer probe will give information about the mass and thereby the density of the asteroid.

\section{Summary}

Bering is a deep space mission to detect and characterize sub-kilometer objects between Jupiter and Venus. The focus on the mission is on the investigation of asteroid evolution, transfer of asteroids from the main belt to the inner Solar System, and determination of meteoritic parenthood. The spacecrafts will carry advanced stellar compasses, a multi spectral imager, a laser ranger and magnetometer probes.

\section{References}

[1] Morbidelli A. \& Nesvorny D. 1999, Icarus 139, 295

[2] Bottke W.F., Cellino A., Paolicchi P. \& Binzel R.P. (eds.), 2002, Asteroids III, University of Arizona Press

[3] Jørgensen J.L. et al., 2003, "MicroASC a miniature star tracker", 4'th International Symposium of the IAA on Small Satellites for Earth Observations, Berlin.

[4] Hansen F. \& Thomsen P.L., 2003, "The Bering mission trade off and scale", these proceedings.

[5] Jørgensen J.L., Denver T., Betto M. \& Jørgensen P.S., 2003, "The Bering target tracing instrumentation", these proceedings.

[6] Davis D.R., Durda D.D., Marzari F., Campo Bagatin A. \& Gil-Hutton R., 2002, in W.F. Bottke, A. Cellino, P. Paolicchi \& R.P. Binzel (eds.) Asteroids III, The University of Arizona Press.

[7] Farinella, P. Vokrouhlicky, D. \& Hartmann, W.K. 1998, Icarus 132,378

[8] Levison H.F. \& Duncan M.J., 1994, Icarus 108, 18

[9] Michelsen R., Haack H., Andersen A.C. \& Jørgensen J.L., "Asteroid and NEA detection models", these proceedings.

[10] Boattini, A. et al. 2003, in preparation

[11] Wetherill, G.W. 1967, J. Geophys. Res. 72, 2429

[12] Jørgensen J.L., Denver T., Betto M. \& Jørgensen P.S., 2003, "The Bering autonomous target detection", these proceedings. 